\title{
Location of Hemorrhage as Predictive Factor for Refractoriness to Blood Pressure Control in Acute, Non-lobar, Hypertensive Intracerebral Hemorrhages
}

\author{
Jose Leonard R. Pascual, ${ }^{1}$ Charisma T. Evangelista ${ }^{2}$ and Jessie T. Colacion ${ }^{3}$ \\ ${ }^{1}$ Department of Anatomy, College of Medicine, University of the Philippines Manila \\ ${ }^{2}$ University of Perpetual Help Dalta Medical Center, Las Piñas City, Philippines \\ ${ }^{3}$ Brunei Neuroscience, Stroke and Rehabilitation Centre, Jerudong Park Medical Centre, Brunei Darussalam
}

\begin{abstract}
Background. Uncontrolled hypertension in acute intracerebral hemorrhages $(\mathrm{ICH})$ may cause hematoma expansion within the first 24 hours, and increase patient mortality. We investigated whether there was an association between ICH location and the difficulty in lowering BP in patients with acute hypertensive nonlobar ICH.
\end{abstract}

Methods. This is a retrospective cohort study of adults diagnosed with non-lobar ICH admitted at a tertiary hospital over a 2-year period. We documented the time to attain target mean arterial pressure (MAP) of $110-130 \mathrm{mmHg}$, as well as the use of antihypertensive medications.

Results. Of 357 patients admitted for non-lobar $\mathrm{ICH}, 47$ patients fulfilled the study criteria. Basal ganglia hemorrhages were the most common (47\%), followed by thalamic (34\%), cerebellar $(11 \%)$, and pontine hemorrhages (8\%). While there were no significant differences in baseline MAP among the different sites of hemorrhage, those with thalamic ICH had a significantly longer time-to-target MAP $(p=0.02)$ and required three or more classes of oral antihypertensive medications $(p<0.001)$.

Conclusions. Acute thalamic intracerebral hemorrhages may require multiple classes of antihypertensives to lower blood pressure to safer levels.

Key Words: intracerebral hemorrhage, refractory hypertension, thalamic, hemorrhage, blood pressure control

\footnotetext{
Corresponding author: Jose Leonard R. Pascual, MD

Department of Anatomy

College of Medicine

University of the Philippines Manila

547 Pedro Gil St., Ermita, Manila, 1000 Philippines

Telephone: +632 5264194

Email: klepnard@gmail.com
}

\section{Background}

Uncontrolled hypertension in acute spontaneous intracerebral hemorrhages ( $\mathrm{SICH}$ ) largely contributes to the continued expansion of hematoma volume within the first 24 hours and leads to increased mortality., ${ }^{1,2}$ Continued hematoma growth up to 6 hours following the initial hemorrhage has been attributed to uncontrolled hypertension and localized coagulopathy. Hematoma volume of greater than $60 \mathrm{~mL}$ as well as initial Glasgow Coma Score (GCS) of 9 or less have been associated with increased mortality. ${ }^{3}$ Current clinical practice guidelines for $\mathrm{SICH}$ recommend careful lowering of the blood pressure (BP) using titratable antihypertensive medications such as labetalol, hydralazine, and nicardipine, while maintaining adequate cerebral perfusion pressure. ${ }^{4}$ Recent trials such as INTERACT and ATACH demonstrated that lowering systolic BP to less than $140 \mathrm{mmHg}$ is safe and reduces risk of hematoma expansion. 5,6

To our knowledge there are no published articles on the relationship between the location of intracerebral hemorrhage and the difficulty of lowering blood pressure in patients with acute hypertensive SICH. It is unclear whether patients with different sites of non-lobar SICH will have the same response to antihypertensive therapy during the first 48 hours of stroke onset. Our aim was to investigate whether there was an association between the location of the hematoma and the difficulty in lowering the blood pressure in patients with acute hypertensive non-lobar intracerebral hemorrhage.

\section{Methods}

This is a retrospective cohort study of patients diagnosed with non-lobar hemorrhagic stroke admitted at a tertiary hospital from January 2006 to December 2007. The study was approved by the hospital's committee on research implementation and development, comprising the technical and ethics review boards. The study included all patients (aged over 45 years), diagnosed with non-lobar intracerebral hemorrhages $(\mathrm{ICH})$ by neuroimaging (cranial computed tomography), and admitted within 48 hours post-ictus, regardless of initial Glasgow coma score (GCS). Patients 
demonstrated to have secondary hypertension and/or bleeding diatheses or coagulopathies were excluded, as well as those with compelling indications for stricter blood pressure control (hypertensive encephalopathy, aortic dissection, acute renal failure, acute pulmonary edema, or acute myocardial infarction). ${ }^{7}$ Cerebral infarcts with extensive hemorrhagic transformation were likewise excluded. Any neurosurgical intervention such as evacuation of hematoma and external ventricular drainage for hydrocephalus was recorded. The hospital records of patients who fulfilled the inclusion criteria and data regarding their co-morbidities was reviewed.

We documented the initial systolic, diastolic, and mean arterial blood pressure recorded at the emergency room as well as the antihypertensive medications administered. Based upon the 2010 guidelines of the American Heart Association and American Stroke Association, adequate BP control was defined as achieving the target MAP of 110-130 mmHg within 48 hours of admission. ${ }^{8}$ We used the following as outcome measures: (1) the time in hours to achieve the target MAP; (2) the use of more than one class of antihypertensive medications to lower the BP; (3) neurosurgical interventions such as external ventricular cerebrospinal fluid drainage or surgical evacuation of the hematoma; and (4) mortality from all causes.

\section{Statistical Analysis}

We used Kruskal-Wallis test for non-normally distributed continuous data such as BP and MAP, and Fisher test for categorical data. Multivariate analysis using linear regression was performed to determine predictors for the time to achieve target MAP. Log rank analysis was also used to determine significant differences in time to target MAP among the different sites of ICH. Statistical significance was set at $\mathrm{p}<0.05$. We used the open source CRAN $\mathrm{R}$ statistical software for our data analysis (http://www.R-project.org/).

\section{Results}

A total of 357 non-lobar hemorrhagic stroke patients aged 46 and above were admitted at a tertiary hospital from January 2006 to December 2007. Ninety-one charts were available and obtained for review, of which 47 patients satisfied the inclusion criteria for the study. Most of these patients were male (64\%). In this study, basal ganglia hemorrhages were most common $(47 \%)$, followed by thalamic $(34 \%)$, cerebellar $(11 \%)$, and pontine hemorrhages $(8 \%)$. This data is comparable to both Western and Asian data regarding the common locations of non-lobar hemorrhages. ${ }^{9,10}$ Table 1 summarizes the demographics of the population in the study. There were no significant differences in the initial hematoma volume among the different sites of $\mathrm{ICH}$ (chi-squared=53.8482, $\mathrm{df}=66, \mathrm{p}=0.86$ ).
The MAP on admission was $128 \pm 21 \mathrm{mmHg}$. There were no significant differences in baseline MAP among the different sites of hemorrhage (Kruskal-Wallis chisquared=21.8637, $\mathrm{df}=21, \mathrm{p}=0.41$ ), and all the patients received intravenous nicardipine infusion titrated to a maximum of $15 \mathrm{mg} /$ hour to achieve the desired MAP of $110-130 \mathrm{mmHg}$. The target MAP was attained within the first 48 hours of admission in $65 \%$ of the patients. The patients with thalamic hemorrhages received nicardipine infusions for a mean duration of 4.4 days, as compared to the patients with basal ganglia (2.3 days), pontine (2.0 days), and cerebellar hemorrhages (1.7 days).

The patients with thalamic hemorrhages had a mean time to target MAP of 93 hours, which was significantly longer than patients with hemorrhages in other areas $(p=0.02)$ (Figure 1). Log rank analysis did not show significant differences in the survival curves of the different locations of $\mathrm{ICH}$ (chi square=3.6, p=0.30, Figure 2). Patients with thalamic hemorrhages were more likely to be on more than two classes of oral antihypertensive medications $(\mathrm{p}<0.001)$ compared with patients with non-thalamic hemorrhages. There was no association between hemorrhage volume and the time to target MAP. The length of time to reach target MAP did not lead to any increase in neurosurgical intervention or mortality. The linear regression analysis is summarized in Table 2.

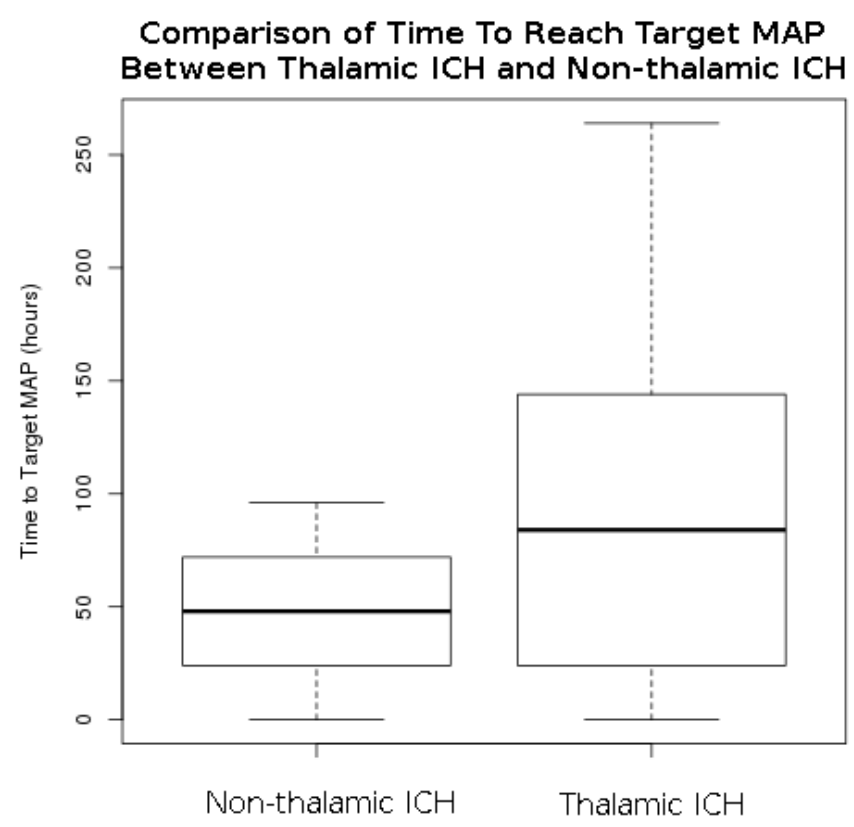

Figure 1. Comparison of Time to Target MAP in Thalamic $\mathrm{SICH}$ vs. Non-Thalamic SICH 
Table 1. Baseline Characteristics

\begin{tabular}{|c|c|c|c|c|}
\hline & $\begin{array}{l}\text { Thalamus } \\
(\mathrm{N}=16)\end{array}$ & $\begin{array}{c}\text { Basal Ganglia } \\
(\mathrm{N}=\mathbf{2 2})\end{array}$ & $\begin{array}{l}\text { Pons } \\
(\mathrm{N}=4)\end{array}$ & $\begin{array}{c}\text { Cerebellum } \\
(\mathrm{N}=5)\end{array}$ \\
\hline Median age (IQR) (years) & $58(55-71)$ & $50(48-56)$ & $56(54-58)$ & $61(60-69)$ \\
\hline Female, $n(\%)$ & $4(25)$ & $7(32)$ & $1(25)$ & $0(0)$ \\
\hline Hypertension, $n(\%)$ & $14(88)$ & $15(68)$ & $4(100)$ & $5(100)$ \\
\hline Diabetes, $n(\%)$ & $0(0)$ & $2(9)$ & $0(0)$ & $2(40)$ \\
\hline Initial Systolic BP, mean, s.d. (mm Hg) & $185.0 \pm 27.3$ & $177.7 \pm 28.4$ & $200.0 \pm 58.9$ & $156.4 \pm 16.3$ \\
\hline Initial Diastolic BP (mm Hg) & $105.3 \pm 16.7$ & $100.9 \pm 17.7$ & $107.5 \pm 38.6$ & $93.6 \pm 11.6$ \\
\hline Initial MAP (mm Hg) & $131.9 \pm 18.7$ & $126.5 \pm 19.2$ & $138.3 \pm 42.6$ & $114.5 \pm 12.9$ \\
\hline Median Glasgow Coma Score & 11 & 14 & 12 & 11 \\
\hline \multirow[t]{2}{*}{ Initial hematoma volume $(\mathrm{mL})$} & $9.8 \pm 7.3$ & $15.9 \pm 9.1$ & $3.0 \pm 1.3$ & $12.2 \pm 6.6$ \\
\hline & $\begin{array}{l}\text { Thalamus } \\
(\mathrm{N}=16)\end{array}$ & $\begin{array}{c}\text { Basal Ganglia } \\
(\mathrm{N}=22)\end{array}$ & $\begin{array}{l}\text { Pons } \\
(\mathrm{N}=4)\end{array}$ & $\begin{array}{c}\text { Cerebellum } \\
(\mathrm{N}=5)\end{array}$ \\
\hline Nicardipine, $n(\%)$ & $11(68)$ & $16(73)$ & $3(75)$ & $3(60)$ \\
\hline Beta blockers, $n(\%)$ & $9(56)$ & $12(54)$ & $4(100)$ & $2(40)$ \\
\hline ACE inhibitors, $n(\%)$ & $11(69)$ & $15(68)$ & $3(75)$ & $3(60)$ \\
\hline Calcium channel blockers, $n(\%)$ & $11(69)$ & $6(27)$ & $3(75)$ & $2(40)$ \\
\hline Angiotensin II receptor blockers, $n(\%)$ & $9(56)$ & $2(9)$ & $0(0)$ & $1(20)$ \\
\hline Single antihypertensive agent, $n(\%)$ & $1(6)$ & $11(50)$ & $0(0)$ & $2(40)$ \\
\hline 2 antihypertensive agents, $n(\%)$ & $4(25)$ & $8(36)$ & $2(50)$ & $3(60)$ \\
\hline$\geq 3$ antihypertensive agents, $n(\%)$ & $11(69)$ & $3(14)$ & $2(50)$ & $0(0)$ \\
\hline
\end{tabular}

Table 2. Linear Regression Analysis for Clinical Endpoints

\begin{tabular}{|c|c|c|c|c|}
\hline & $\begin{array}{c}\text { More than } 3 \text { antihypertensive } \\
\text { class of drugs }\end{array}$ & $\begin{array}{c}\text { More than } 48 \text { hrs to } \\
\text { target MAP }\end{array}$ & $\begin{array}{c}\text { Underwent } \\
\text { Neurosurgery }\end{array}$ & Mortality \\
\hline Age & $1.00(0.98-1.03)$ & $0.99(0.97-1.01)$ & $0.99(0.98-1.00)$ & $1.00(0.99-1.02)$ \\
\hline Female gender & $0.92(0.54-1.56)$ & $0.89(0.63-1.26)$ & $1.68(0.47-5.94)$ & $1.09(0.76-1.56)$ \\
\hline DM & $0.51(0.21-1.25)$ & $0.87(0.49-1.56)$ & $0.86(0.66-1.13)$ & $0.94(0.51-1.71)$ \\
\hline Initial GCS & $1.06(0.97-1.16)$ & $0.98(0.92-1.04)$ & $0.93(0.91-0.96)^{* * *}$ & $0.95(0.89-1.01)$ \\
\hline Volume of ICH & $1.00(0.97-1.03)$ & $0.99(0.97-1.01)$ & $0.99(0.98-1.00)$ & $1.00(0.97-1.02)$ \\
\hline \multicolumn{5}{|l|}{ Location of ICH } \\
\hline Thalamus & $\begin{array}{c}3.06(1.76-5.30)^{* * *} \\
2.65(1.01-6.97)\end{array}$ & $0.70(0.49-1.01)$ & $2.15(0.53-8.68)$ & $0.96(0.66-1.40)$ \\
\hline Pons & $1.23(0.54-2.78)$ & $0.56(0.30-1.06)$ & $1.46(0.33-6.39)$ & $1.36(0.71-2.60)$ \\
\hline Cerebellum & & $1.37(0.81-2.33)$ & $1.43(1.12-1.83)^{* *}$ & $0.89(0.52-1.55)$ \\
\hline
\end{tabular}

$$
\text { *** } \mathrm{p}<0.001
$$$$
\text { ** } \mathrm{p}<0.01
$$

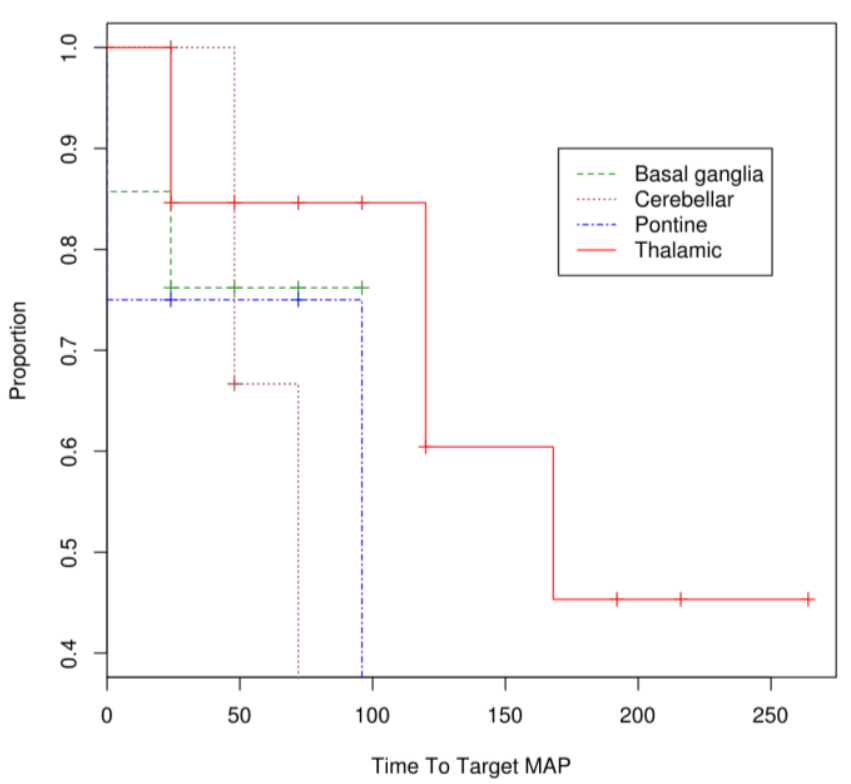

Figure 2. Survival Curve of SICH Patients' Time to Target Mean Arterial Pressure

\section{Discussion}

Patients with acute thalamic hemorrhages may have markedly refractory hypertension requiring combinations of antihypertensive drugs on top of intravenous nicardipine. The refractoriness of hypertension in this subset of hypertensive intracerebral hemorrhages may be due to increased sympathetic drive in the nearby diencephalic structures such as the hypothalamus. The paraventricular nucleus (PVN) of the hypothalamus has been shown to be the master controller of the autonomic nervous system functions, ${ }^{11}$ with spinally projecting pre-autonomic neurons $(\mathrm{SPAN})^{12}$ that target the cardiovascular system. Either increased stimulation by excitatory neurotransmitters or loss of GABA-mediated inhibition of the PVN may lead to increased heart rate and hypertension. ${ }^{13-21}$ One experimental study showed that the overexpression of diencephalic thyroliberin (dTRH) induced arterial hypertension in rats. ${ }^{22}$ Thalamic hemorrhages with compression upon the hypothalamus have been shown to present clinically with miosis by disrupting the descending sympathetic fibers. ${ }^{23}$ It may be possible that other homeostatic mechanisms of the 
hypothalamus may be disrupted by such compression, leading to increased sympathetic drive and elevated blood pressure.

\section{Conclusions}

There is an association between the thalamic site of intracerebral hemorrhage and difficulties in lowering markedly elevated blood pressures during the first 48 hours of admission. While the patients with thalamic intracerebral hemorrhages did not differ in baseline blood pressure and MAP from the patients with non-lobar hemorrhages at other sites, this subset of patients took significantly longer and needed more than two classes of antihypertensives to lower the $\mathrm{BP}$ to the target MAP. The findings of this study may impact future clinical trials investigating the efficacy of BP lowering in the acute phase of $\mathrm{SICH}$, as patients with thalamic hemorrhages may have increased sympathetic drive and may require combinations of several classes of oral antihypertensive medication to achieve the target mean arterial or systolic blood pressures.

\section{Limitations and recommendations}

As this was a retrospective study with low chart retrieval rates, the sample sizes for each group of $\mathrm{ICH}$ site was small, particularly for the pontine and cerebellar hemorrhages. Although the selection of oral antihypertensive drug classes was based on existing clinical practice guidelines during the study period, the administration of the drugs may have been inconsistent and dependent on the patients' financial status.

We recommend further research into the control of hypertension in acute thalamic hemorrhages using a prospective cohort design, with a larger sample size for each location of non-lobar intracerebral hemorrhage. A prospective study would allow standardization in the dosing and titration of intravenous antihypertensives such as nicardipine, as well as the utilization of the different classes of oral antihypertensive medications. We also recommend the addition of clinical endpoints of hematoma expansion as well as the development of worsening perilesional edema, to further delineate the importance of refractoriness of hypertension in ICH patients.

\section{References}

1. Ohwaki K, Yano E, Nagashima H, Hirata M, Nakagomi T, Tamura A. Blood pressure management in acute intracerebral hemorrhage: relationship between elevated blood pressure and hematoma enlargement. Stroke. 2004;35(6):1364-7.

2. Willmot M, Leonardi-Bee J, Bath PM. High blood pressure in acute stroke and subsequent outcome: a systematic review. Hypertension. 2004; 43(1):18-24.

3. Broderick JP, Brott TG, Duldner JE, Tomsick T, Huster G. Volume of intracerebral hemorrhage: a powerful and easy-to-use predictor of 30 day mortality. Stroke. 1993; 24(7):987-93.
4. Broderick JP, Adams HP Jr, Barsan W, et al. Guidelines for the management of spontaneous intracerebral hemorrhage: a statement for healthcare professionals from a special writing group of the Stroke Council, American Heart Association. Stroke. 1999; 30(4):905-15.

5. Anderson CS, Huang Y, Wang JG, et al. Intensive blood pressure reduction in acute cerebral haemorrhage trial (INTERACT): a randomised pilot trial. Lancet Neurol. 2008; 7(5):391-9.

6. Antihypertensive Treatment of Acute Cerebral Hemorrhage (ATACH) investigators. Antihypertensive treatment of acute cerebral hemorrhage. Crit Care Med. 2010; 38(2):637-48.

7. Kaplan NM. Management of hypertensive emergencies. Lancet. 1994; 344(8933):1335-8.

8. Morgenstern LB, Hemphill JC 3rd, Anderson C, et al. Guidelines for the management of spontaneous intracerebral hemorrhage: a guideline for healthcare professionals from the American Heart Association/American Stroke Association. Stroke. 2010; 41(9):2108-29.

9. Boonyakarnkul S, Dennis M, Sandercock P, Bamford J, Burn J, Warlow C. Primary intracerebral haemorrhage in the Oxfordshire Community Stroke Project. Cerebrovasc Dis. 1993; 3(6):343-9.

10. Zafar A, Khan FS. Clinical and radiological features of intracerebral haemorrhage in hypertensive patients. J Pak Med Assoc. 2008; 58(7):3568 .

11. Spyer KM. Annual-review prize lecture - central nervous mechanisms contributing to cardiovascular control. J Physiol (London). 1994; 474(1):1-19.

12. Coote JH. Landmarks in understanding the central nervous control of the cardiovascular system. Exp Physiol. 2007; 92(1):3-18.

13. Nunn N, Womack M, Dart C, Barrett-Jolley R. Function and pharmacology of spinally-projecting sympathetic pre-autonomic neurones in the paraventricular nucleus of the hypothalamus. Curr Neuropharmacol. 2011; 9(2):262-77.

14. Badoer E, Ng CW, De Matteo R. Tonic sympathoinhibition arising from the hypothalamic PVN in the conscious rabbit. Brain Res. 2002; 947(1):17-24.

15. Martin DS, Haywood JR. Hemodynamic responses to paraventricular nucleus disinhibition with bicuculline in conscious rats. Am J Physiol. 1993; 265(5 Pt 2):H1727-33.

16. Martin DS, Segura T, Haywood JR. Cardiovascular responses to bicuculline in the paraventricular nucleus of the rat. Hypertension. 1991; 18(1):48-55.

17. Kannan H, Hayashida $Y$, Yamashita H. Increase in sympathetic outflow by paraventricular nucleus stimulation in awake rats. Am J Physiol. 1989; 256:(6 Pt 2):R1325-30.

18. Schlenker E, Barnes L, Hansen S, Martin D. Cardiorespiratory and metabolic responses to injection of bicuculline into the hypothalamic paraventricular nucleus (PVN) of conscious rats. Brain Res. 2001; 895 (12):33-40.

19. Li YF, Wang W, Mayhan WG, Patel KP. Angiotensin-mediated increase in renal sympathetic nerve discharge within the PVN: role of nitric oxide. Am J Physiol-Regul Integr Comp Physiol. 2006; 290(4):R1035-43.

20. Martin DS, Haywood JR. Sympathetic nervous-system activation by glutamate injections into the paraventricular nucleus. Brain Res. 1992; 577(2):261-7.

21. Martin DS, Haywood JR, Thornhill JA. Stimulation of the hypothalamic paraventricular nucleus causes systemic venoconstriction. Brain Res. 1993; 604(1-2):318-24.

22. Burgueño AL, Landa MS, Schuman ML, et al. Association between diencephalic thyroliberin and arterial blood pressure in agouti-yellow and ob/ob mice may be mediated by leptin. Metabolism. 2007; 56(10):1439-43.

23. Kumral E, Kocaer T, Ertubey NO, Kumral K. Thalamic hemorrhage: a prospective study of 100 patients. Stroke. 1995; 26(6):964-970. 